\title{
Mature teratoma with aspergilloma
}

\author{
Abebe Bezabih', Asfaw Atnafu² \\ 1. Department of Surgery, School of Medicine, College of Health Sciences, Addis Ababa University, Addis Ababa, Ethiopia \\ 2. Department of Radiology, School of Medicine, College of Health Sciences, Addis Ababa University, Addis Ababa, Ethiopia
}

Correspondence: Dr Abebe Bezabih (bezieabe@yahoo.com)

(ㅇ) 2018 A. Bezabih \& A. Atnafu. This open access article is licensed under a Creative Commons Attribution 4.0 International License (http://creativecommons.org/licenses/ by/4.0/), which permits unrestricted use, distribution, and reproduction in any medium, provided you give appropriate credit to the original author(s) and the source, provide a link to the Creative Commons license, and indicate if changes were made.

East Cent Afr J Surg. 2018 Dec;23(3):119-122 https://dx.doi.org/10.4314/ecajs.v23i3.6

\begin{abstract}
Mediastinal mature teratomas are benign germ cell tumors which rarely involve the lung, but when they invole the lung they can cavitate. Aspergilloma developing in a mature teratoma is extremely rare, and according to our English literature search, there is only one previously reported case. We report a 21 -year-old female who presented with cough and foul-smelling sputum. investigations revealed an intrathoracic mass, which intraoperatively and upon subsequent histological exam was found to be a mature mediastinal teratoma involving the lung and associated with an aspergilloma. Reporting our case will add to the understanding of this rare presentation of mediastinal mature teratomas.
\end{abstract}

Keywords: mature teratoma, aspergilloma, surgical treatment

\section{Introduction}

Mediastinal mature teratomas are germ cell tumors that can affect the lung ${ }^{1-3}$ and the pericardium and can cause diagnostic dilemma unless there is high index of suspicion.

Like other pulmonary cavitary lesions that can be complicated by the formation of an aspergilloma, mature teratomas can lead to a similar complication. However, aspergilloma associated with a mature teratoma is extremely rare, and our English-language literature review has revealed only one previous similar case report to ours, which follows below. ${ }^{3}$

\section{Case presentation}

\section{History}

A 21-year-old female patient from Somali Region, Filtu area, Ethiopia, presented with cough and foul-smelling (occasionally blood-tinged) profuse sputum of 18 months duration. The amount of sputum was about half a liter per day. She also had mild shortness of breath especially on walking uphill and exercise. She also complained of right-sided chest pain but had no fever or weight loss. She was seen in Filtu rural hospital one year and six months back where she was put on anti-tuberculosis therapy for 6 months but showed no improvement.

\section{Physical examination}

The patient was comfortable and not in distress. Vital signs: pulse rate $-86 / \mathrm{min}$ regular, blood pressure - 100/60 $\mathrm{mmHg}$, respiratory rate -18 breaths per minute, axillary temperature- 36 degrees Celsius. Examination of the head, ear, nose, and throat, lymphoglandular, and the cardiovascular systems showed normal findings. Respiratory system examination showed symmetric chest moving with respiration. There was comparatively increased tactile fremitus, dullness, and decreased air entry over the right posterior middle lung field. Otherwise, the rest of the physical examination was normal.

\section{Diagnostic assessment}

Complete blood count, liver and renal function tests were all within normal range at admission. Tests of tumor markers showed normal levels of carcinoembryonic antigen, alpha-fetoprotein, and CA-125 but slightly elevated levels of beta human chorionic gonadotrophin. Ultrasound of the abdomen was normal.

Chest x-ray showed homogenous lobulated right lower lung opacity, which appeared larger compared to the scout film of the chest CT (Figure 1). The mass had apparently low density areas (white star, Figure 1B) and loss of visualization of the right cardiac border. Right lateral film confirmed the anterior location of the mass.

Chest CT scan done on 28 th July 2016 showed a $112 \times 75$ $\times 81 \mathrm{~mm}$ intrathoracic cavitating mass with thick irregular heterogeneously enhancing walls and a focal defect in the inferior wall (Figure 2). The cavitary lesion contained multiple rounded masses arising from its internal wall. Some of the masses had -75 to $-118 \mathrm{HU}$, fat density (arrow 3A) with no contrast enhancement except peripherally, while some were 

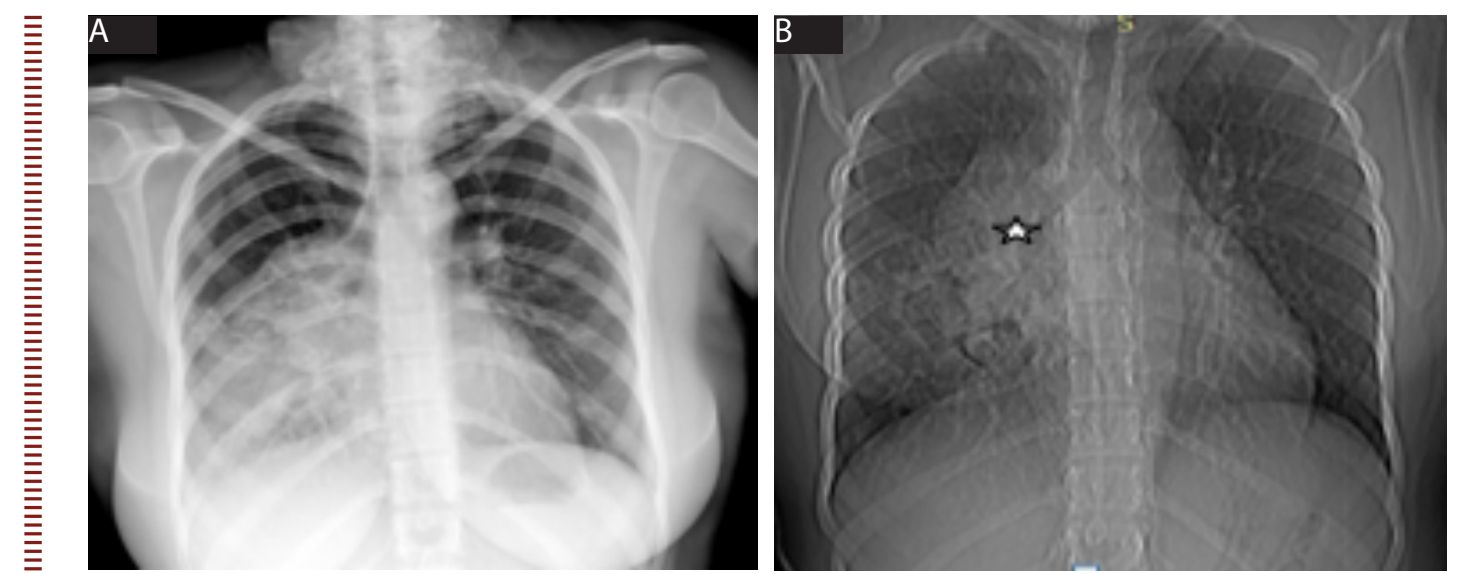

Figure 1. Chest xray (a) done 20 after the CT scout film(b) showed homogenous lobulated right lower lung opacity which hasan apparently low density areas (white arrow $b$ ) and loss of visualization of the right cardiac border
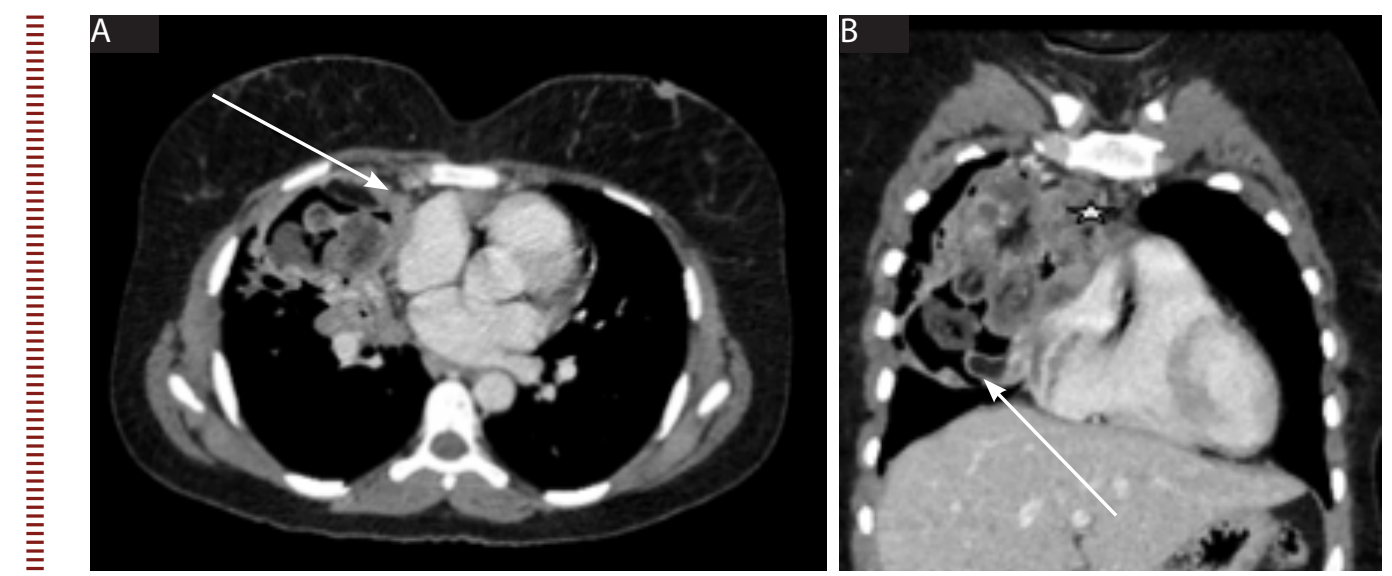

Figure 2. (A) axial, and (B) coronal chest CT scan of the patient which showed anterior Mediastinal cavitating mass with thick irregular heterogeneously enhancing wall (arrow A) with focal defect in its inferior wall (arrow B)
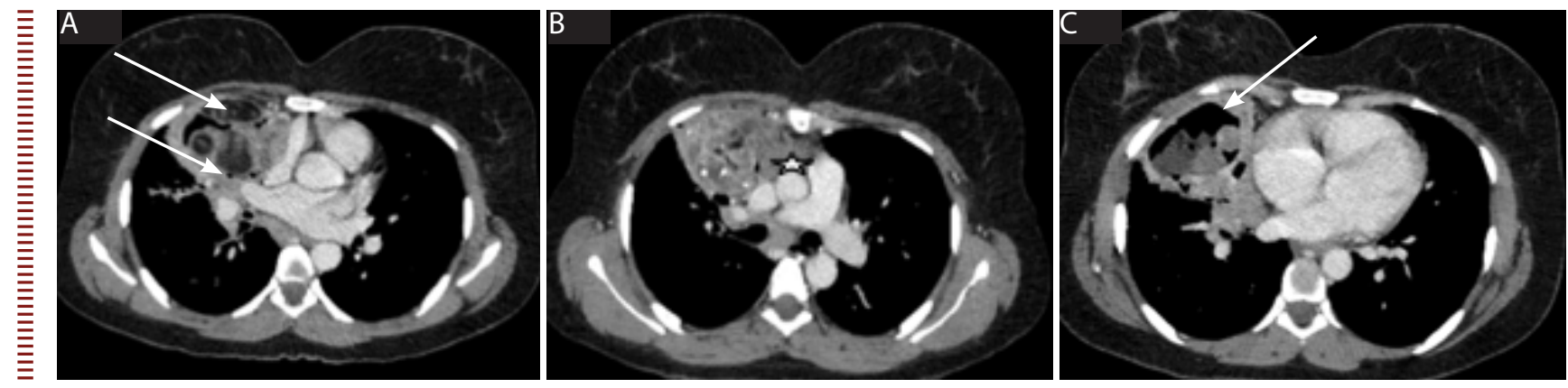

Figure 3. (A), (B), and (C) axial post contrast CT chest scan of the patient at different level showed fat density rounded density masses (arrow A), heterogeneously enhancing solid soft tissue masses (Figure 2B and 3B, white star) with calcification (3B) and rounded well-defined soft tissue masses located around the inferior wall of the cavity (arrow C)

heterogeneously enhancing solid soft tissue masses (Figures $2 \mathrm{~B}$ and $3 \mathrm{~B}$, white star ). Additionally, there were also rounded, well-defined soft tissue masses located around the inferior wall of the cavity (Figure 3C). Multiple amorphous calcifications were also seen (Figure 3B).

The mass extended to the lung, anterior chest wall and mediastinal wall and compressed the vascular structures, including the afferent superior vena cava at its entrance (Figures $2 \mathrm{~B}$ and $3 \mathrm{~A}$ ) and the right atrium. The right main pulmonary artery at the hilum were surrounded by the mass. No fat plane was observed between the mass and the surrounding structures. Alveolar infiltrates were seen around the lesion in the lung window.

Overall imaging findings suggested a heterogeneous solid cavitating anterior mediastinal mass with signs of rupture, calcification, and varying density masses internally. Such findings radiologically suggested a mature benign teratoma. However the presence of a heterogeneous mixed-density pattern suggested malignancy. 


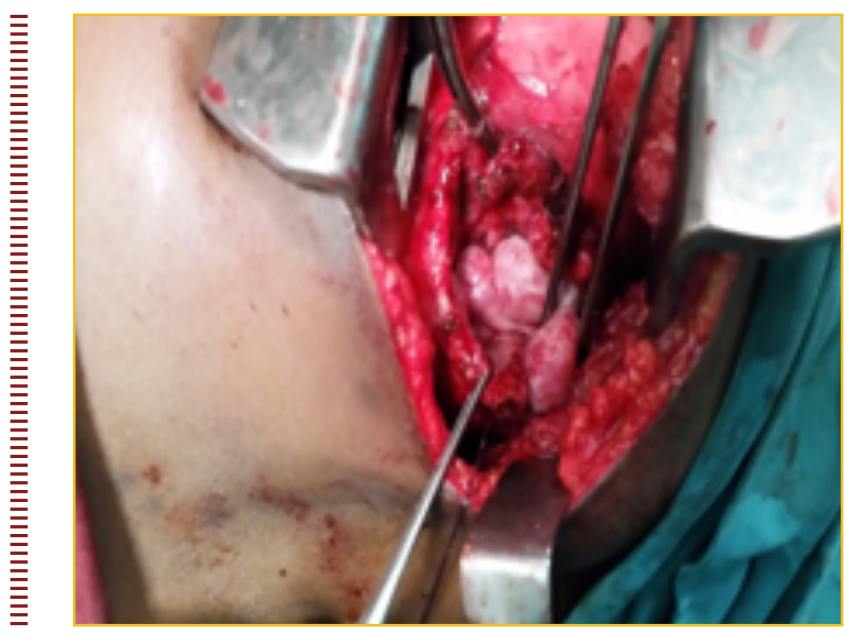

Figure 4. intraoperative picture showing multiple polyps inside a cavity at the right upper lobe of the lung.

\section{Intervention}

The patient was admitted to the Tikur Anbassa Specialised Hospital Department of Surgery on August 15, 2016, where she underwent surgery. Through a right posterolateral thoracotomy, the chest cavity was entered and the findings were a mass originating from the mediastinum involving the right upper lobe of the lung. There was a cavity at the center of the mass communicating with open bronchi. Inside the cavity was an aspergilloma (Figure 4 ). The mass had involved the right phrenic nerve inseparably and was adherent to the pericardium, which was separated with difficulty. The cavity was entered and the aspergilloma evacuated. Then the mass was dissected off the right upper lobe and peeled off the pericardium, but the right phrenic nerve could not be separated from the mass and was sacrificed. The cavitary mass with part of the phrenic nerve were removed. The bronchial holes on the right upper lobe were closed with polypropylene sutures. The chest was closed after leaving a chest tube. The patient was sent to the intensive care unit intubated and stayed two days in the intensive care unit, after which she was extubated safely. The patient was discharged from hospital on 14 September 2016 without any complications.

The resected specimen was sent for pathologic examination. The gross specimen was $17 \mathrm{~cm}$ in diameter, light grey mixed with brown, firm, and containing multiple polyps and hair shafts (Figure 4). The cut surface showed yellowish and whitish solid components with areas of small cystic spaces filled with serous (clear) fluid.

Microscopy showed a variety of tissues including adipose tissue (Figure 5B), keratinized squamous epithelium and hair follicles (Figure 5E), sweat and sebaceous glands (Figure $5 \mathrm{~A}$ ), as well as intestinal and pancreatic tissues (Figure 5C, 5D), and aggregates of lymphatic tissue. These findings are suggestive of mature teratoma.

\section{Follow up and outcomes}

The patient was re-evaluated as an outpatient three weeks after surgery. She had no shortness of breath, no cough, no fever, and no other complaints. On physical examination, the chest was resonant and clear to auscultation. She had smooth postoperative course up to that poing and was scheduled for follow-up one month later. A final check was done 7 weeks after surgery, and the patient again had no complaints. She was comfortable and physical examination showed normal vital signs, as well as a clear and resonant chest. Control chest $\mathrm{x}$-ray showed good bilateral lung expansion with no evidence of residual lesion or recurrence.
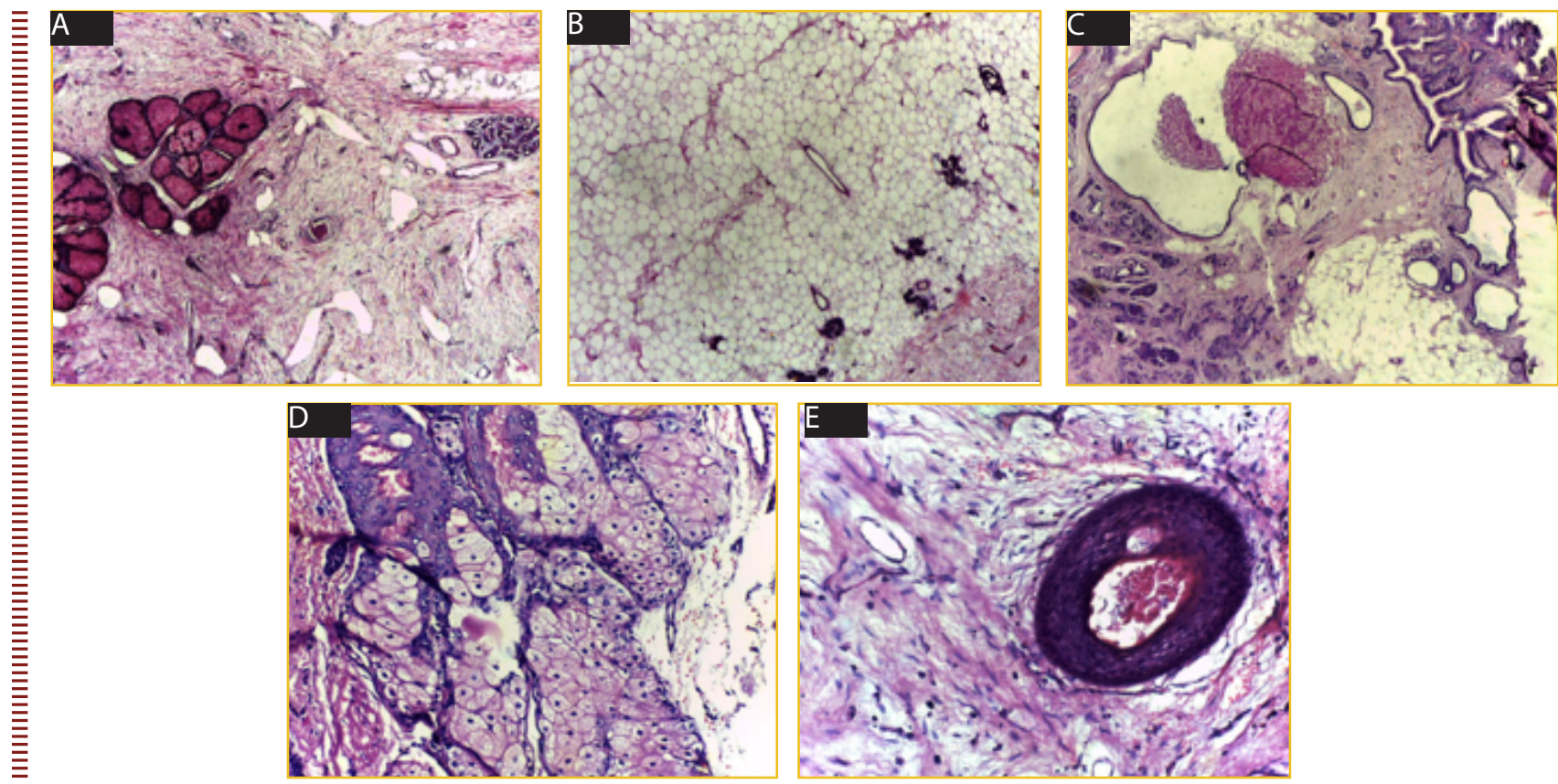

Figure 5. Histologic images showing a variety of tissues including skin appendages(derivatives of ectoderm), adipose tissues (derivatives of mesoderm), and intestinal tisssues and pancreas(derivatives of endoderm). (A) Sweat glands, sebaceous glands and dilated thin walled vessels; (B) adipose tissue; (C) intestinal epithelium admixed with pancreatic tissue in fibrous background; (D) intestinal epithelial lining; (E) a hair follicle 


\section{Discussion}

Teratomas are germ cell tumors arising from pluripotent embryonic cells, and the anterior mediastinum is a commonly affected site. ${ }^{2,6} \mathrm{CT}$ is an important imaging modality, and the finding of combination of fluid, fat, calcification, and soft tissue attenuation in an anterior mediastinal mass is highly specific for a teratoma and is helpful in distinguishing it from other common anterior mediastinal tumors, such as thymomas and lymphomas. ${ }^{7-9}$ The surrounding pressure, inflammation, or digestive enzymes which can appear in this condition may erode and rupture the mass to the adjacent structures and cause cavitation. It is known that a preexisting pulmonary cavity can be colonized by fungi and can potentially form a fungal ball. The cause for cavitary lung diseases can be variable and include diseases like tuberculosis, sarcoidosis, tumors, pulmonary fibrosis, bronchiectasis, or histoplasmosis. Even though a mature mediastinal teratoma ruptured to the lungs with communication to bronchi can theoretically be colonized by fungi, to our knowledge and as per our search of English literature (PubMed and HINARI), there has only been one previously reported case of an aspergilloma growing in a mature teratoma. ${ }^{3}$

The cause of involvement of surrounding structures like the phrenic nerve and surrounding lung by a mature teratoma, a benign tumor, might be pressure necrosis causing erosions, inflammatory changes, and fibrosis.

Our CT finding of heterogeneous solid cavitating anterior mediastinal mass with signs of rupture, calcification, and varying density mass internally is typical for mature teratoma (Figures 2 and 3). The radiologic report did not suggest the presence of a superimposed aspergilloma. We suggest that the rounded, well-defined soft tissue density masses which were located around the inferior wall of the cavity and had different attenuation behavior should raise the index of suspicion of this rare pathological mix.

The intraoperative findings were confirmatory of an aspergilloma, which was removed from the cavity manually and by suction with the teratoma, which consisted of finger-like polypoid projections in to the cavity (Figure 4). Bronchial holes were also communicating with the cavity confirming rupture (invasion) of the mass into the right upper lobe of the lung. The communication of the tumor's cavity with open bronchi explains the growth of the aspergilloma, as Aspergillus species are ubiquitous aerophilic fungi with an affintiy for growth in the lung cavities. Histologic examination of the operative specimen confirmed the diagnosis of mature mediastinal teratoma.

\section{Conclusions}

In conclusion, our case described a rare condition, aspergilloma in a mature teratoma. Preoperative diagnosis either clinically or based on imaging is difficult. Clinicians and radiologists must have an index of suspicion for this rare pathological mix. Particularly, the finding of unusual round soft-tissue masses with a different CT attenuation and enhancing pattern should hint at the possibility of teratoma colonized by a fungus.

\section{Acknowledgements}

This work would have been impossible had it not been for the consent given by our patient to use all the information provided. We also would like to acknowledge the Pathology Department of Tikur Anbassa Specialized Hospital for cooperating with the release of gross and microscopic features of the patient's result.

\section{Competing interests}

Both authors declare that they have no competing interests related to this work.

\section{References}

1. Otah Y, Sato H, Seki M, Endo M, Tsubota M.; A case of anterior mediastinal mature teratoma containing air because of penetration of the lung. Nihon Kyobu Geka Gakkai Zasshi; 1993 Jun, 41(6);1114-8.

2. Boussetta K, Jaziri F, Bousnina D, Aloui N, Meherzi A, Kilani T, KammounSellami N, Bousnina S. A propos of a mediastinal teratoma invading the lung: Arch pediatr, 2001 Dec; 8(12):1344-7.

3. Kobayashi T, Yamamoto H, Ohtsuka T, Jmura Y.; Resection of a mediastinal teratoma that perforated the lung and combined with aspergilloma in the tumor cyst; Nihon Kyobu Geka Gakkai Zasshi 1992 Nov; 40(11):20914

4. Tsukamoto S, Omori K, Kitamura K, Ohata M, Sezai Y, Nemoto N.; A case report of mediastinal teratoma complicated with cardiac tamponade: Nihon Kyobu Geka Kakkai Zasshi 1993 Apr 41(4):688-93.

5. Ooman A, Santhosham R, Vijayakumar C, Ramachandran P, Kumar S.; Anterior mediastinal teratoma presenting as cardiac tamponade: Indian Heart J, 2004 Jan - Feb; 56(1):64-6.

6. Iwasaki $T$, luchi $K$, Matsumura A, Sueki $H$, Yamamoto $S$, Mori $T$. Intrapulmonary mature teratoma. Jpn J ThoracCardiovasc surg. $2000 \mathrm{Jul}$ 48(7):468-72.

7. Pikin O, Kolbanov K, Kazakevich V, Korolev A. ; Mediastinal mature cystic teratoma perforating into the lung: Interact Cardiovasc Thorac Surg 2010 Dec; 11(6):827-9.

8. Nakajima K, Yamada G, Tanaka H, Saizen H, Naqata M, Tanaka s, Itoh T Koba H, Abe S, Sato M. A case of combined squamous cell carcinoma and aspergilloma arising in a cyst wall. Nihon Kokyuki Gakkai Zasshi 2001 Dec;39(12):961-5.

9. Shojiroh Morinaga, Hiroaki Nomori, Ryichiro Kobayashi, Yoshihito Atsumi. ; Well-differentiated adenocarcinoma arising from mature cystic teratoma of the mediastinum -reporting a surgical case. Ajcp, Apr 1994, 101(4):531-4.

10. Sakamoto K, Kase M, Mo M, Kurata $H_{\text {.; }}$ Mediastinal mature teratoma perforated in to the pericardial sac; a case report.:KyobuGeka 2000 Jan; 53(1):74-7.

11. Lee M, Lee Y, Ikusima H, Chin S, Lee M, Sakurai M. ; Resection of mediastinal teratoma in a patient with cardiac tamponade due to pericardial perforation: Nihon Kyobu Geka Gakkai Zasshi 1991 Sep; 39(9):1771-6.

12. Yanq CJ, Chenq YJ, Kang WY, Huang MS, Hwang JJ.; A case of dermoid cyst ruptured into the lung. Respirology 2007 Nov; 12(6):931-3.

13. David J. Revere, Amgad N. Makaryus, Elias P. Bonaros, L. Michael Graver.; Chylopericardium presenting as cardiac tamponade secondary to an anterior mediastinal cystic teratoma: Tex Heart Inst J 2007 ;34:379-82.

14. Seval Gunes, Joseph Varon, CarrettWalsch.; Mediastinal teratoma presenting as massive hemoptysis in an adult- case report: Journal of emergency medicine, May-June 1997 Vol 15(3):313-6. 